\title{
Number of Node Estimation in Mobile Ad Hoc Networks
}

\author{
https://doi.org/10.3991/ijim.v11i6.6986 \\ Saher Manaseer $\left.{ }^{\square}\right)$, Ibrahem Alhabash \\ The University of Jordan, Amman, Jordan \\ sahereju.edu.jo
}

\begin{abstract}
Mobile Ad hoc networks do not have any form of centralized control as per their definition. Therefore, many challenges face the development of efficient protocols for such networks. One of the main challenges is the difficulty of extracting the total number of nodes in the network, this paper aims to find an estimate for this number using the average hop count for messages exchanged in the network. Experiments of this paper are conducted on Ad Hoc networks under DSR and AODV routing protocols. Results show a relation between network size and average hop count that can be used to predict the number of nodes in the network.
\end{abstract}

Keywords-Ad hoc, MANETs, AODV, DSR, Node count, Simulation, NS2, Topology.

\section{Introduction}

Recently, Mobile Ad hoc Networks (MANETs) are considered a significant challenge. The significant attention they have gained via the easiness of setup and usability in many fields [1]. Yet many challenges arise to control the topology especially the hidden or neglected parts of these infrastructure-less networks [2].

Wireless networks have emerged in the $1970 \mathrm{~s}$, then they were used to enable mobility[1]. MANETs began in the 1990s as a special type of Ad Hoc Networks. The difference was in the node mobility. In the mid of 1990, MANETs became popular and formed a significant research topic due to the emergence of $802.11 / \mathrm{Wi}$ Fi wireless networking, PDAs and the laptops [1][3][4].

The flexibility that enables MANETs to be used in many fields gave them their importance and the attention they have received [4]. Disasters recovery, security and military operations, are examples where MANETs considered significant [2]. A node in a MANET, can perform as a router, not only sender and receiver for data [5].

The dynamic nature of infrastructure-less networks such as MANETs, makes it important to find the routing paths and control the energy limitation problems, which have become significant issues [6],[7]. MANETs are used in situations where there is no need for a control unit because they are self-configured, through deployment of the nodes that can collectively set a network [8], [9].

This paper aims at detecting the number of active nodes in a network using DSR and AODV routing protocols. A better knowledge of the number of operating nodes 
or transmitters present in the network area is a major factor that supports the proper operation of the network. [10]

Sometimes it could be useful to know or at least estimate the number of active nodes composing the network, for example in parameter estimation or in nonparametric regression [11]. In a distributed functionality protocol, number of nodes cannot be extracted using centralized methods. The estimation of the number of active agents is important also for peer-to-peer networks [11]. Moreover, knowledge of number of nodes is useful in the case of topology analysis [12]. knowledge of the total number of nodes in a network, even an estimated number is useful in developing efficient protocols for MANETs. It has been reported in the leteratuer that, knowledge of the total number of nodes in a MANET is one of the major missing factors that might affect protocol design decisions [10]. For instance, the number of nodes is a factor that affects the choice of speed in many mobility models [10] For example, estimating the total number of nodes can improve the media access control protocol.

\section{Related works}

In real life, any mobile device is considered a node in a network. Traces and synthetic models are the main types of mobility models used in network simulation [13]; in the case of large mobile nodes number traces are used, for they provide accurate and adequate information. Traces are the patterns observed and found in real life. The other type is synthetic models that is used to represent how the mobile nodes acts in a realistic way [14], [15]. The synthetic models contain entity and group mobility models, the entity models represent the nodes that act and move independently of each other [16].

Number of nodes in a networks affects the decision that the simulation process, such as the type of protocol, routing, etc. Thus, detecting the number of nodes in a network has become a concern for researcher [5].

In [17], Kim et al. have found that the number of nodes that are transmitting data in parallel in the wireless network can be estimated by using clustering techniques. Their proposed method to analyzes the data of the Received signal strength indicator (RSSI) as a based parameter of a network, and The link quality indicator (LQI), which is less affected by the environment than the RSSI, and reveals the quality of the received signal between nodes. The information extracted from analyzing the RSSI and LQI data indicate the number of active nodes that are transmitting data in parallel in a nearby network. However, the results were not reliably correct since the classification was unsupervised.

Moreover, the RSSI measurement depends on other factors than number of sending nodes. Such factors are transmission range and battery life.

In addition, knowing the number of nodes can support some setup conditions. For example, a low density network allows scenarios with higher speeds of mobility [18].

To make sure that the network covers the targeted area, an optimization for the number of nodes should be considered. In an experiment to do achieve that Using NS2 simulator, Kim et al in [17] have found that the network should contain at least three 
times the commonly known minimum necessary nodes to gain a stable connection for a simulation. Simulation parameters should be analyzed to measure the performance of the network, such as the size of the area that the network covers and number of nodes, since the number of the nodes affects the output [8], [9]. On the other hand, the features and characteristic of the network such as dynamicity of the topology and the transmission medium, can affect the security and reliability of connection [19], [20]. This highlights how the estimation of node number in the active network is essential and can affect the steadiness of the connection [21].

\section{Experiment Results and Analysis}

DSR and AODV routing protocols are used in this experiment to achieve desired outcomes.

The Dynamic Source Routing protocol (DSR) is an efficient protocol that is widely used in Ad hoc networks because it enables the existence of network to be selfconfigured without the infrastructure. It is composed mainly of two techniques; the routing discovery and the routing maintenance which works collaboratively to find the paths, maintain and preserve routes for destinations on the network [22].

The Ad hoc On-Demand Distance Vector (AODV) routing protocol, provides quick readjustment and adaptation to the connection conditions, such as the low processing and utilization of the network. It uses destination nodes sequence to guarantee loop-free routes most of the time [18].

In this paper, the two protocols were used to find an estimation for the number of nodes using the average hop count. The use of these two protocols serves the target of extracting the number of nodes in the network with the minimum possible overhead. In the limited resource environment present in a MANET, overheads have significant impact on performance levels and life times.

\subsection{Simulation Parameters}

In this experiment, the parameters run for both AODV and DSR are:

Table 1. parametes selected in this experiment

\begin{tabular}{|l|l|}
\hline \multicolumn{1}{|c|}{ Parameter } & \multicolumn{1}{c|}{ Value } \\
\hline Area & $1000 * 1000$ \\
\hline Time & $800 \mathrm{~s}$ \\
\hline Nodes & From $10-140$ \\
\hline Minimum speed & 1 and maximum 4 \\
\hline Transmission range & $100,125,150,175,200,250$ meter \\
\hline
\end{tabular}

The results extracted from simulation confirm the relation between number of nodes and average hop count. It is a logical consequence that routes become longer when increasing the number of nodes. However, the relation is expected. 
The main goal of this research is to find an estimation for the number of nodes using the average hop count. Figure 1 demonstrates the average hop count for DSR protocol using transmission range of 250 meters. It can be seen that for network size of 10 to 70 nodes, the average hop count is 1 .

The value increases for sizes between 80 and 120 to reach 2 and then increases to 3 when the network size is above 120. Although the resolution is not high for the first range, this value usable to estimate the number of nodes in an Ad Hoc network. Moreover, the estimation is easier for larger network sizes. The same conclusion is valid for other transmission ranges as the figures 2, 3 and 4 show.

The figure indicates that the hop count is not a strong indicator when the network size is small, less than 80 nodes in this case. This could be affected by many factors such as mobility patterns, initial node location and clustering in the internet.

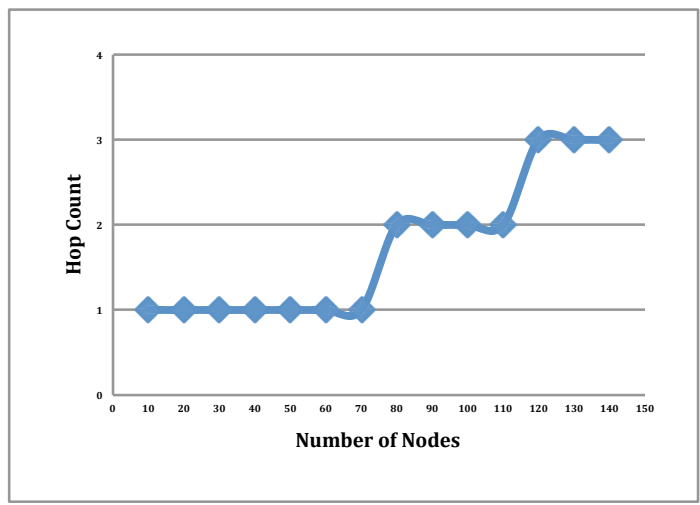

Fig. 1. Average Hop Count for DSR against number of nodes at transmission range of $250 \mathrm{~m}$

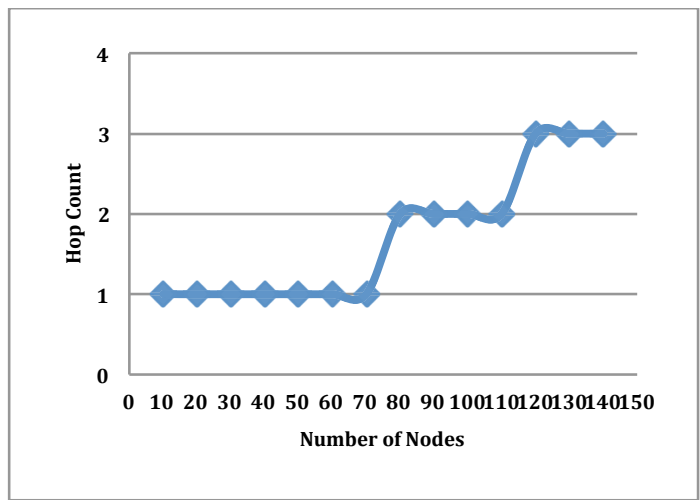

Fig. 2. Average Hop Count for DSR against number of nodes at transmission range of $200 \mathrm{~m}$ 


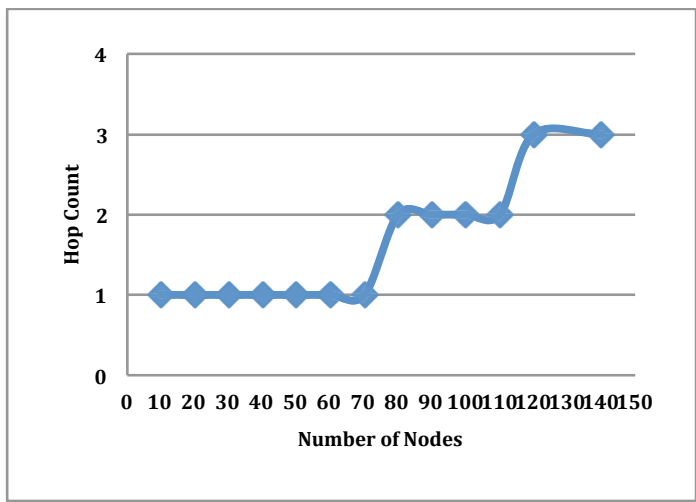

Fig. 3. Average Hop Count for DSR against number of nodes at transmission range of $150 \mathrm{~m}$

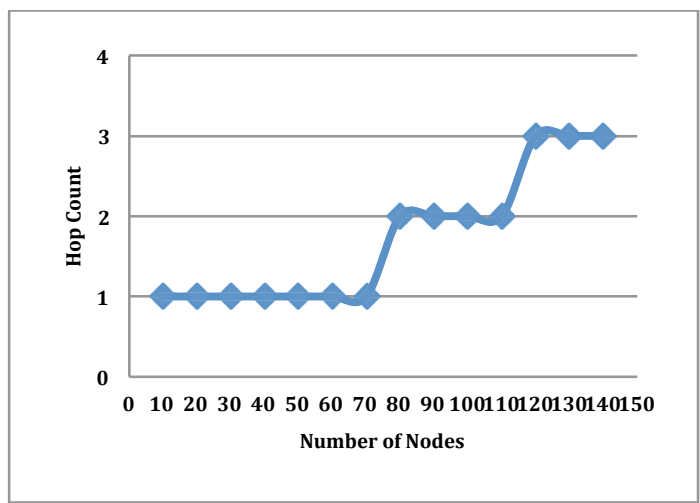

Fig. 4. Average Hop Count for DSR against number of nodes at transmission range of $100 \mathrm{~m}$

As for AODV, the results offer higher resolution and a clear relation between the number of nodes and the average hop count. Moreover, average hop count is more sensitive to increasing transmission range.

Therefore, the suggested estimation is more suitable for Ad Hoc networks that use AODV. The relation is clear in the Figures 5, 6, 7 and 8 below.

It is demonstrated that results for small network sizes are also not strong indicators. However, the results for AODV show better resolution under 80 nodes compared to DSR results. This difference indicated that in addition to the factor mentioned earlier, the routing protocol is also a factor that affects the relation between average hop count and total number of nodes. 


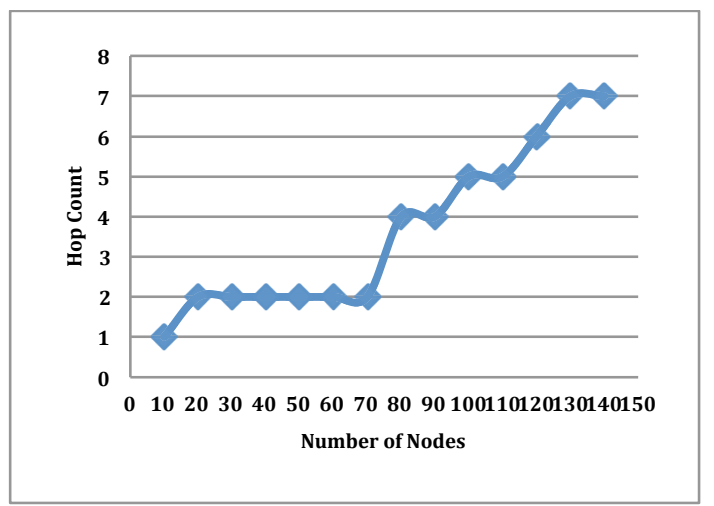

Fig. 5. Average Hop Count for AODV against number of nodes at transmission range of $250 \mathrm{~m}$

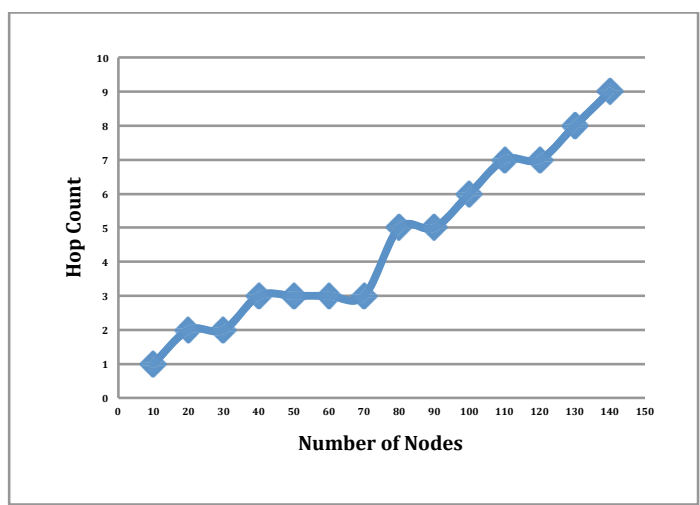

Fig. 6. Average Hop Count for AODV against number of nodes at transmission range of $200 \mathrm{~m}$

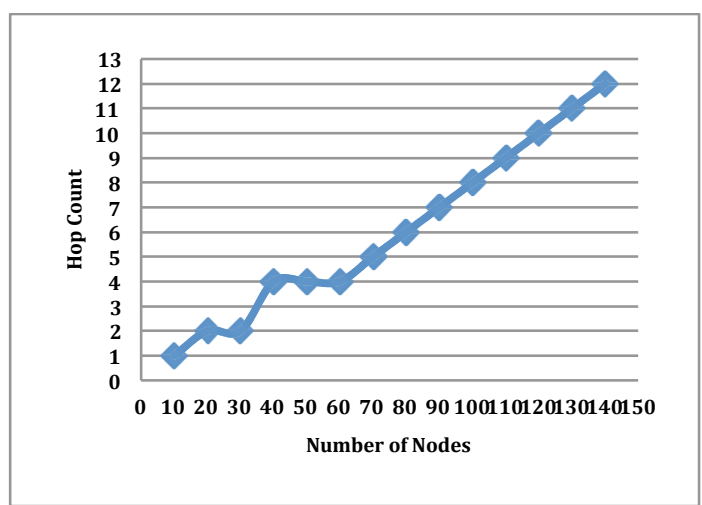

Fig. 7. Average Hop Count for AODV against number of nodes at transmission range of $150 \mathrm{~m}$ 


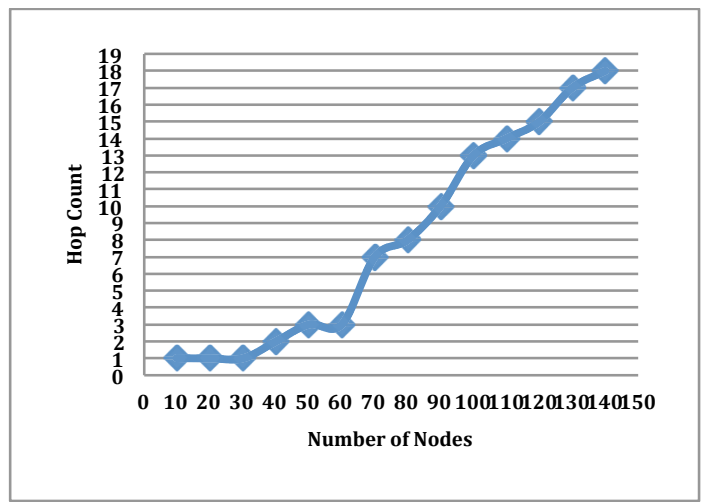

Fig. 8. Average Hop Count for AODV against number of nodes at transmission range of $100 \mathrm{~m}$

\section{Conclusion}

This paper has introduced the usage of hop count of messages in estimating the total number of nodes in mobile Ad hoc networks under ADOV and DSR routing protocols. Results have shown the relation between the two factors and the applicability of this estimation. Moreover, results have revealed that the relation is stronger under ADOV such that the resolution of estimates is higher than it is in the case of DSR. The results also show that the relation between hop count and node numbers is not of high resolution for small network sizes.

\section{$5 \quad$ References}

[1] Manaseer, S. (2010). On backoff mechanisms for wireless mobile ad hoc networks. Diss. University of Glasgow,

[2] Eze, Elias C., et al. (2016). Advances in vehicular ad-hoc networks (VANETs): Challenges and road-map for future development." International Journal of Automation and Computing 13.1:1-18. https://doi.org/10.1007/s11633-015-0913-y

[3] Bassant, S. and Yeob Yeun, C. (2015 ). Key management for the MANET: A survey." Information and Communication Technology Research (ICTRC), International Conference on. IEEE.

[4] Omari, M., Zohra, K., and Hasna. S. (2016). Simulation Comparison and Analysis Of Network Throughput In DSDV And AODV And DSR Mobile Ad hoc Network Protocols. African Review of Science, Technology and Development 1.02.

[5] Manaseer, S. (2016). On the Choice of Parameter Values for Simulation Based Experiments on Mobile Ad Hoc Networks. International Journal of Communications, Network and System Sciences, 9(04), 90. https://doi.org/10.4236/ijens.2016.94008

[6] Kim, Y. et al. (2007). The number of necessary nodes for ad hoc network areas." AsiaPacific Service Computing Conference, the 2nd IEEE. IEEE, 2007. 11-14. https://doi.org/10.1109/APSCC.2007.60

[7] Mahgoub, I., and Ilyas. M., (2016). Sensor network protocols. CRC press. 
[8] Jewel, D., et al. (2016). Improved Hole Detection Healing and Replacing Algorithm for Optimal Coverage in Wireless Sensor Networks.

[9] Feng, Z., and J. Guibas, L. (2004). Wireless sensor networks: an information processing approach. Morgan Kaufmann,.

[10] Md Shamim, A., Frater, M., and J. Ryan. M. (2009). Estimation by cross-correlation of the number of nodes in underwater networks. Telecommunication Networks and Applications Conference (ATNAC), Australasian. IEEE..

[11] Damiano, V., Pillonetto, G., and Schenato. L. (2010). Distributed statistical estimation of the number of nodes in sensor networks. Decision and Control (CDC), 2010 49th IEEE Conference on. IEEE..

[12] Manaseer, S., Asoudi, D., and Aljawawdeh, A. (2017). Border Node Detection: A New experimental approach. Computer Modelling and New Technologies, Volume 12, 2017

[13] John, T., Dotche, K. A., and Diawuo. K. (2012). The Impact Of Mobile Nodes Arrival Patterns In Manets Using Poisson Models. International Journal of Managing Information Technology 4.3 (2012): 54.

[14] Sourabh, G., and Singh. A. (2016). Extensive Survey on Mobile Ad-Hoc Network Detection and Prevention. International Journal of Engineering Science 4877.

[15] Alamgir, S. et al. (2016). Mobile Ad hoc networks in the Global system of Interconnected Computer Networks. IJSEAT 4.9: 434-451.

[16] Aniket, P., et al. (2016). Behavioral study of Random Waypoint Mobility Model based energy aware MANET. Signal Processing and Integrated Networks (SPIN), 2016 3rd International Conference on. IEEE.

[17] Wang, Y., Guardiola, I, and Wu, X (2014), "RSSI and LQI data clustering teqniques to determine the number of nodes in wireless sensor networks", International Journal of distributed sensor networks, V 2014, ID 380526.

[18] John, T., Diawuo, K., and A. Dotche.K. (2012). The Effect Of The Number Of Mobile Nodes On Varying Speeds Of MANETS. arXiv preprint arXiv:1212.2567.

[19] Khamayseh, Y., Bader, A., Mardini, W., and Yasein, B. (2011), "A new protocol for detecting black holes nodes in Ad Hoc networks", International Journal of Communication Networks and Information Security, Vol 3. No1.

[20] Pimal, K. and Trivedi. B (2017). Security in Mobile Ad Hoc Networks. Proceedings of International Conference on Communication and Networks. Springer, Singapore.

[21] Ali, G. (2006). Vulnerability and Security of Mobile Ad hoc Networks, Proceedings of the $6^{\text {th }}$ WSEAS International Conference on Simulation, Modelling and Optimization, Lisbon, Portugal, September 22-24.

[22] Johnson, D. and Maltz, D. (2007). The Dynamic Source Routing Protocol (DSR) for mobile Ad Hoc Networks for IPv4. Network Working Group/ Experimental. Carnegie Mellon University.

\section{Authors}

Saher Manaseer and Ibrahem Alhabash are with The University of Jordan, Amman, Jordan (saher@ju.edu.jo).

Article submitted 08 April 2017. Published as resubmitted by the authors 23 May 2017. 